\title{
Load Sharing in Human-Robot Cooperative Manipulation
}

\author{
Martin Lawitzky, Alexander Mörtl and Sandra Hirche \\ Institute of Automatic Control Engineering \\ Technische Universität München \\ 80290 Munich, Germany \\ Email: \{ml,moertl,hirche $\}$ tum.de
}

\begin{abstract}
Physical cooperation with humans greatly enhances the capabilities of robotic systems when leaving standardized industrial settings. In particular, manipulation of bulky objects in narrow environments requires cooperating partners. Actuation redundancies arising in joint manipulation impose the question of load sharing among the interacting partners. In this paper, effort sharing policies are systematically derived from the geometric and dynamic task properties. Three policies are intuitively identified, resulting in unilateral and balanced effort distributions. These policies are evaluated within a novel hierarchical motion generation and control framework. The synthesized system is successfully validated in a threedegrees-of-freedom planar tracking experiment. This evaluation shows an interdependency of the load sharing strategy and the resulting task performance.
\end{abstract}

\section{INTRODUCTION}

Perfect automation of all different kinds of physical tasks in daily life, industrial applications, offices and construction sites is still a distant prospect. However, one can imagine that robots will soon be able to execute a much broader variety of tasks in cooperation with humans. While speech and gestures in such a setting are used to negotiate tasks and goals, haptic interaction is characterized by the simultaneous negotiation and accomplishment of tasks that require physical coupling. This special feature makes haptic humanrobot interaction a particularly challenging research topic. In this paper we will focus on the joint manipulation of a rigid object under environmental constraints. Two important challenges in this context are: i) As soon as two or more partners jointly manipulate one object, redundancies allow an efficient shaping of the load distribution by the partners. So far it is unclear, which load sharing policy should be applied by the robotic partner. ii) Dextrous handling of an object in a constrained environment requires explicit consideration of the object's shape and dynamics. In the context of load sharing human-human experiments have shown that indeed specialized strategies emerge in terms of temporarily consistent haptic interaction patterns [1]. This role distribution among the partners might be the key factor to the repeatedly observed improved task performance in cooperative settings [2], [3]. Recent work aims to synthesize robot behaviors based on these findings [4], [5]. Assuming a predefined plan given to a human-robot dyad, a leaderfollower controller blending framework for a robot partner enables a continuously adjustable interactive behavior of both partners [4]. Trajectories from human-human experiments are successfully replicated using this method. Yet, no

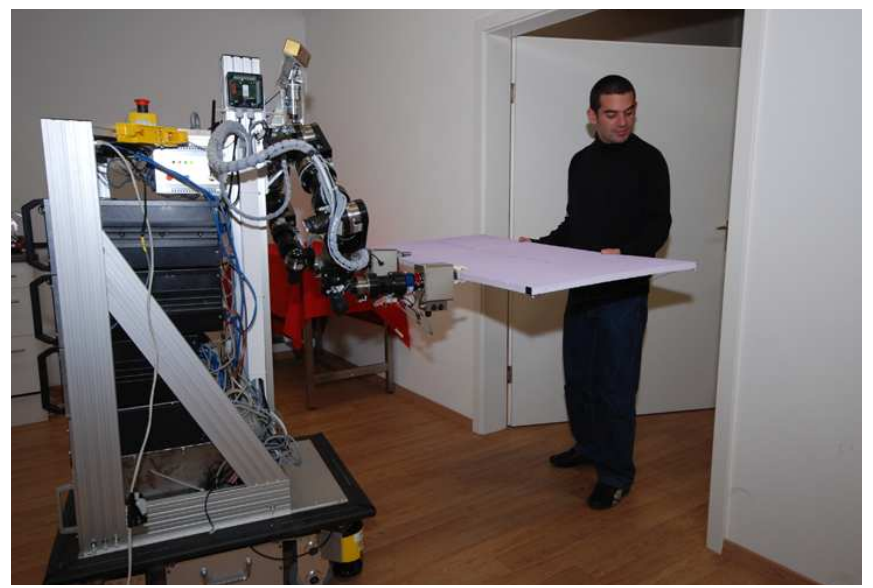

Fig. 1. Envisaged scenario of a joint load transport: Human and robot cooperatively carry a bulky object through a constrained environment.

concrete approach for the setting of the blending factors is given. A dynamic role-based effort sharing scheme utilizing force thresholds on known user force profiles leads to a subjective cooperation improvement, yet no quantitative enhancement [5]. In their current formulation, both synthesis approaches [4], [5] do not consider spatially distributed grasp points which are required for the manipulation of bulky objects. The problem of manipulation of bulky objects by multiple robots is addressed in [6] proposing a decentralized control architecture, which however cannot be applied in the presence of human partners. Constraint-based approaches for joint human-robot manipulation of bulky objects yield effective task-specialized solutions [7], [8]. To the best of our knowledge, no explicit load sharing beyond gravity compensation by the robot partner has been considered. Caster-like robot partner behavior reactively compensating the object dynamics is well-suited for human-robot joint bulky load transport as shown in [9], [10]. Such a follower strategy for the robot may fail, however, in constrained environments where the robot must actively contribute to the object's configuration trajectory. Furthermore, the results from human-human experiments on improved task performance through cooperation motivate us to aim at similarly effective load sharing strategies for human-robot cooperation.

The contribution of this work is a systematic derivation of effort sharing policies from a system-theoretic analysis of the joint object manipulation task under environmental constraints. The task of joint load transportation is con- 
sidered cooperative since we assume willing participation of all agents towards a common goal following a shared plan [11], [12]. The load sharing policies are parameterized with respect to their degree of assistance. As a result, the physical load is dynamically shared depending on the task state and the assistance degree. By this parameterization the proactivity of the robot, i.e. how actively it advances the task, can be tuned. The derived policies are experimentally evaluated in a novel hierarchical architecture for motion generation and control. The experimental evaluation shows an interdependency of the load sharing strategy and the resulting task performance.

The remainder of this paper is organized as follows: After the problem statement and a system-theoretic analysis of the joint load transport task is given in section II, the theory is applied to an illustrative planar task in section III and a set of effort sharing policies is derived. A planning and control architecture is proposed in section IV. The paper concludes after an experimental evaluation in section $\mathrm{V}$.

Notation: By convention, bold characters are used for vectors and matrices. $\operatorname{Ker}(\boldsymbol{A})$ denotes the kernel or nullspace of matrix $\boldsymbol{A} . \operatorname{Ker}_{j}(\boldsymbol{A})$ denotes the $j$-th vector spanning $\boldsymbol{A}$ 's nullspace. A matrix's nullity is the dimension of its nullspace. We further adopt the convention of placing orientation variables (angular position, velocity and acceleration, and torque) before the translational variables (linear position velocity, acceleration, and force). The configuration space of the manipulated rigid object is denoted $\mathcal{C}$ which is a threedimensional manifold $\mathcal{C}=\mathbb{R}^{3} \times \mathbb{R} \mathbb{P}^{3}$. The obstacle region is denoted $\mathcal{C}_{\text {obs }} \subseteq \mathcal{C}$. The leftover configurations are called free space which is denoted $\mathcal{C}_{\text {free }}=\mathcal{C} \backslash \mathcal{C}_{\text {obs }}$.

\section{System-Theoretic AnAlysis of the Joint Manipulation TASK}

Our work addresses the cooperative task of joint manipulation of a rigid bulky object with multiple humans and robots. The demand for an effort sharing strategy arising from actuation redundancy is of special interest. In this context, effort sharing describes the distribution of a desired force input among the human and robot agents.

We confine our problem to the following conditions:

- One or more humans cooperate with one or more robots in the sense of a common known goal: The final configuration of a commonly manipulated object.

- All participants tightly grasp the same rigid object with commonly known shape and dynamics.

- No other than haptic interaction via the object among the collaborating human-robot team is possible.

- Environmental constraints are such that a feasible path to the goal exists.

In the following, a system-theoretic analysis will give further insights to the generalized problem of joint object manipulation under environmental constraints. The need for a planning and motion-generation strategy will be shown and a parameterized effort sharing policy is systematically derived.

\section{A. Dynamical System Model}

The dynamical model of the object is described by

$$
\boldsymbol{M}_{c} \ddot{\boldsymbol{x}}_{c}+\boldsymbol{D}_{c} \dot{\boldsymbol{x}}_{c}=\boldsymbol{u}_{c}
$$

where $\boldsymbol{x}_{c}$ is the configuration of the object with inertia $\boldsymbol{M}_{c}$ and viscous friction $\boldsymbol{D}_{c} ; \boldsymbol{u}_{c}$ denotes the external wrench on the object. In this work, the general case for multiple humans

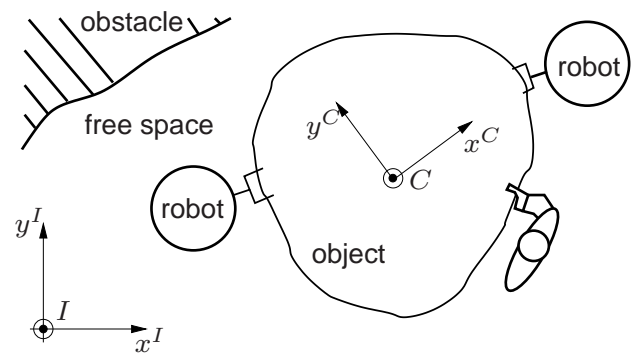

Fig. 2. Haptic human-robot interaction task: Cooperative manipulation of a rigid object by multiple agents under environmental constraints.

and robots - at least two - is described. We consider $N$ multiple robot manipulators and $M$ multiple human arms rigidly holding a common object as shown in Fig. 2. An object frame $C$ is attached to the load, the world frame is denoted by $I$.

At the grasp point, agent $i$ applies wrench $\boldsymbol{u}_{i}$. Selection matrices $\boldsymbol{S}_{i}$ determine which torques and forces can be applied at the grasp point. Matrix $\boldsymbol{S}_{i}$ reduces the input wrench $\boldsymbol{u}_{i}$ to an effective applied wrench

$$
\tilde{\boldsymbol{u}}_{i}=\boldsymbol{S}_{i} \boldsymbol{u}_{i}
$$

Thus, the external wrench on the object is composed by

$$
\boldsymbol{u}_{c}=\sum_{i=1}^{M+N} \boldsymbol{J}_{i c}^{T} \tilde{\boldsymbol{u}}_{i}
$$

Matrix $\boldsymbol{J}_{i c}$ denotes the $\left(\operatorname{dim}\left(\tilde{\boldsymbol{u}}_{i}\right) \times \operatorname{dim}\left(\boldsymbol{u}_{c}\right)\right)$-Jacobian of the kinematic constraint $\phi_{i}\left(\boldsymbol{x}_{c}\right)$. Constraint $\phi_{i}\left(\boldsymbol{x}_{c}\right)$ is the position of the rigid grasp point w.r.t. the object frame. Thus, for the position $\boldsymbol{x}_{i}$ of the grasp point of agent $i=1, \ldots, M+N$ we can derive

$$
\begin{aligned}
& \boldsymbol{x}_{i}=\boldsymbol{\phi}_{i}\left(\boldsymbol{x}_{c}\right) \\
& \dot{\boldsymbol{x}}_{i}=\boldsymbol{J}_{i c} \dot{\boldsymbol{x}}_{c} .
\end{aligned}
$$

\section{B. System Properties}

In order to show the need for proactive robot behavior under environmental constraints, i.e. $\mathcal{C}_{\text {obs }} \neq \emptyset$ for such a system, we derive relevant properties of the dynamical system and their requirements from a system-theoretic point of view:

1) Controllability: Controllability represents an important property of a dynamical system, as it describes the ability to move the system state from any initial state to any final state in finite time using the external inputs [13]. In contrast to our problem stated above, controllability does not comprise any (environmental) constraints on the system state. Controllability under state constraints is only analyzed for the very limited set of right-invertible linear systems [14] but not for our non-linear problem. If we include obstacles in 
the configuration space, i.e. $\mathcal{C}_{\text {obs }} \neq \emptyset$ and therefore the statespace of the considered system, it is straightforward to show that a certain desired final state may not be reachable though the system is controllable in a control-theoretic sense, see e.g. Fig. 3. Controllability related to our addressed problem

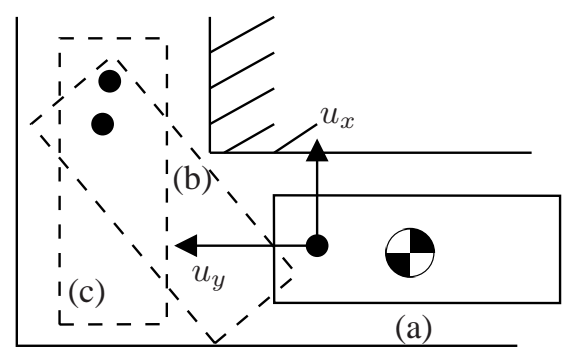

Fig. 3. Variable direction thruster example of a controllable system, yet unable to arrive at desired state due to environmental constraints. (a) is the initial configuration, (b) the required - but not reachable - intermediate configuration and (c) the desired final configuration.

is thus a necessary but not sufficient condition to accomplish the cooperative task.

2) Holonomicity: As an even stronger system property describing concisely our needs we use the term of holonomicity, meaning a system does not have any velocitydependent i.e. non-integrable constraints. Concretely, such a system can be instantly accelerated in an arbitrary direction of the configuration space $\mathcal{C}$. Holonomicity is a sufficient condition for the feasibility of our considered task.

3) Redundancy: Closely connected to the holonomicity condition, we require at least the same number of nonredundant control inputs as corresponding configuration variables [15]. In case of redundancy regarding the control inputs to the system, the problem of effort sharing has to be paid special attention. Note that the robotic agents could decide how many manipulators $N$ are required to fulfill the task for a given number $M$ of human inputs.

\section{Effort Sharing}

In the following, we discuss the degrees of freedom for effort sharing, arising from actuation redundancy. Assuming a commonly known trajectory of the desired object configuration $\boldsymbol{x}_{c, d}$, we aim for solutions of the agent's applied wrench $\tilde{\boldsymbol{u}}$. Given $\boldsymbol{x}_{c, d}$ the desired external wrench $\hat{\boldsymbol{u}}_{c}$ can be easily computed for the resulting holonomic system using the inverse dynamical system model. The external wrench is the part of the applied wrench $\tilde{\boldsymbol{u}}$ that causes the object's motion. The remainder of the applied wrench is called the internal wrench or squeeze wrench. Since the external wrench is composed by the applied wrenches, we can write

$$
\boldsymbol{M}_{c} \ddot{\boldsymbol{x}}_{c}+\boldsymbol{D}_{c} \dot{\boldsymbol{x}}_{c}=\boldsymbol{J}^{T} \boldsymbol{A} \hat{\boldsymbol{u}}_{c}
$$

where $\boldsymbol{A}$ denotes the mapping from desired external wrenches to applied wrenches so that

$$
\tilde{\boldsymbol{u}}=\boldsymbol{A} \hat{\boldsymbol{u}}_{c}
$$

the stacked transposed Jacobian matrix $\boldsymbol{J}^{T}$ is composed by

$$
\boldsymbol{J}^{T}=\left[\begin{array}{lll}
\boldsymbol{J}_{1, c}^{T} & \cdots & \boldsymbol{J}_{M+N, c}^{T}
\end{array}\right] .
$$

Note that $\operatorname{dim}\left(\hat{\boldsymbol{u}}_{\boldsymbol{c}}\right)$ is equal to the dimension of the object's configuration space $\operatorname{dim}\left(\boldsymbol{x}_{c}\right)$. In our setting we assume that

$$
\operatorname{dim}(\tilde{\boldsymbol{u}})>\operatorname{dim}\left(\hat{\boldsymbol{u}}_{\boldsymbol{c}}\right)
$$

holds. The number of actual inputs is larger than the required number of inputs for task completion requiring effort sharing policies in the redundant application envisaged. In consequence, the selection of matrix $\boldsymbol{A}$ is not unique. In fact, it describes the solution of the effort sharing problem. In the following, we will examine a possible composition of matrix $\boldsymbol{A}$ explicitly providing a parameterization for effort sharing strategies. The generalized Moore-Penrose pseudoinverse $\left(\boldsymbol{J}^{T}\right)^{+}$of the stacked transposed jacobian matrix $\boldsymbol{J}^{T}$ is an option for the effort-sharing matrix $\boldsymbol{A}$ that will be investigated here. It yields the minimum-norm solution for $\|\tilde{\boldsymbol{u}}\|$ and thus has no null-space component $\operatorname{Ker}\left(\boldsymbol{J}^{T}\right)$ [16] defined as

$$
\operatorname{Ker}\left(\boldsymbol{J}^{T}\right)=\left\{\tilde{\boldsymbol{u}} \mid\left(\boldsymbol{J}^{T}\right) \tilde{\boldsymbol{u}}=\mathbf{0}\right\} .
$$

Note, that the physical meaning of the null-space component is the squeeze wrench applied to the object causing no motion. Replacing $\boldsymbol{A}$ by $\left(\boldsymbol{J}^{T}\right)^{+}$in (3), the family of solutions for $\tilde{\boldsymbol{u}}$ is given by

$$
\tilde{\boldsymbol{u}}=\left(\boldsymbol{J}^{T}\right)^{+} \hat{\boldsymbol{u}}_{\boldsymbol{c}}+\sum_{j=1}^{\operatorname{nullity}\left(\left(\boldsymbol{J}^{T}\right)\right)} \lambda_{j} \operatorname{Ker}_{j}\left(\boldsymbol{J}^{T}\right)
$$

This decomposition into object motion-causing and squeeze forces imposes the problem of choosing $\lambda_{j}$.

\section{AnAlysis OF EFFORT SHARING POLICIES}

For intuitiveness of the analysis and the subsequent effort sharing synthesis approach we consider from this section on a planar cooperative manipulation scenario involving two agents. The example scenario with one human and one robot aiming at the joint transport of a bulky object is depicted in Fig. 4.

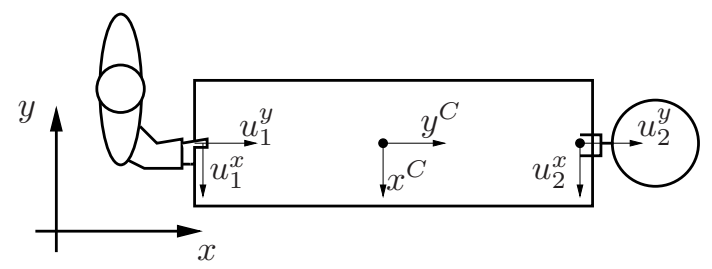

Fig. 4. Planar scenario of cooperative manipulation.

Imagine a large table on caster wheels, satisfying the holonomicity condition. Generally, both human and the robot partner can apply forces in the two-dimensional plane as well as torques around their grasp points. Thus, we can write for $\boldsymbol{u}$

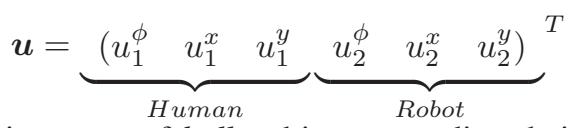

The main aspect of bulky objects regarding their handling is the lack of sensitivity to applied torques at the grasp points. Using this approximation, the selection matrix $S$ reduces the input vector to dimension 4 neglecting applied torques at the grasp points

$$
\tilde{\boldsymbol{u}}=\left(\begin{array}{c}
u_{1}^{x} \\
u_{1}^{y} \\
u_{2}^{x} \\
u_{2}^{y}
\end{array}\right)=\left(\begin{array}{llllll}
0 & 1 & 0 & 0 & 0 & 0 \\
0 & 0 & 1 & 0 & 0 & 0 \\
0 & 0 & 0 & 0 & 1 & 0 \\
0 & 0 & 0 & 0 & 0 & 1
\end{array}\right) \boldsymbol{u}
$$


The kinematic constraints of the system can be expressed as

$$
\boldsymbol{x}_{i}^{I}=\boldsymbol{x}_{c}^{I}-\boldsymbol{R}_{i, c}^{C}=\phi_{i}\left(\boldsymbol{x}_{c}\right)
$$

with

$$
\boldsymbol{R}=\left(\begin{array}{cc}
\cos \phi & -\sin \phi \\
\sin \phi & \cos \phi
\end{array}\right)
$$

denoting the rotation of $C$ with respect to $I$ and $\boldsymbol{r}_{i, c}^{C}$ being the vector from the grasp point of agent $i$ to the origin of $C$. This leads to the $4 \times 3$ jacobian matrix

$$
\boldsymbol{J}=\left(\begin{array}{ccc}
s_{\phi} r_{1, c}^{(x)}+c_{\phi} r_{1, c}^{(y)} & 1 & 0 \\
-c_{\phi} r_{1, c}^{(x)}+s_{\phi} r_{1, c}^{(y)} & 0 & 1 \\
s_{\phi} r_{2, c}^{(x)}+c_{\phi} r_{2, c}^{(y)} & 1 & 0 \\
-c_{\phi} r_{2, c}^{(x)}+s_{\phi} r_{2, c}^{(y)} & 0 & 1
\end{array}\right)
$$

Using (1), (2) and (5), the resulting dynamical system can be written as

$$
\boldsymbol{M}_{c} \ddot{\boldsymbol{x}}_{c}+\boldsymbol{D}_{c} \dot{\boldsymbol{x}}_{c}=\boldsymbol{J}^{T}\left(\begin{array}{llll}
u_{1}^{x} & u_{1}^{y} & u_{2}^{x} & u_{2}^{y}
\end{array}\right)^{T} .
$$

Controllability of this non-linear system is proven for even one two-dimensional control input $\tilde{\boldsymbol{u}}=\left(\begin{array}{cc}u_{1}^{x} & u_{1}^{y}\end{array}\right)^{T}$ applied anywhere on the object except the center of gravity [13]. Obviously, controllability cannot get lost by adding a second control input. Bullo and Lewis proposed motion planning algorithms for the single-input case [17]. However, such algorithms do not allow for obstacles. Note that we can calculate for our given planar system

$$
\forall \phi \quad \operatorname{dim}\left(\boldsymbol{x}_{c}\right)=\operatorname{rank}(\boldsymbol{J})=3,
$$

i.e. we can apply holonomic motion planning methods. Before investigating the effort sharing policies we will discuss the optimal grasp points.

\section{A. Optimal Selection of Grasp Points}

We can derive straightforwardly the optimal selection of grasp points from (6) w.r.t. the system's manipulability. Here we consider a system's manipulability measure $\mu$ which is defined as

$$
\mu=\sqrt{\operatorname{det}\left(\boldsymbol{J}^{T} \boldsymbol{J}\right)} .
$$

After straightforward calculation of (7) using (6), one finds

$$
\mu=\sqrt{2}\left\|\boldsymbol{r}_{1, c}-\boldsymbol{r}_{2, c}\right\|
$$

Maximizing the value of $\mu$ minimizes the applied wrenches at the grasp points for a given external wrench $\boldsymbol{u}_{c}$ by maximizing the Jacobian's eigenvalues in (2). This leads to the intuitive result that the partners should grasp the object with maximum distance. Note, that this optimization is only valid for bounded $\boldsymbol{r}_{i, c}$ and does not explicitly weigh angular vs. translational motion.

\section{B. Effort Sharing Policies}

As shown in section II-C, the system's redundant force inputs can be used to share parts of the task effort among the agents according to different criteria. Such effort sharing can be either dynamic in terms of changing roles or static. In a first step we will investigate static sharing policies in the following. Note, that static policies refer to constant roles in cooperation, not to static load distributions, depending on the task.
Recalling the synthesis of the agent's force inputs $\tilde{\boldsymbol{u}}$ from (4), in our example application the kernel $\operatorname{Ker}\left(\boldsymbol{J}^{T}\right)$ is spanned by the family

$$
\operatorname{Ker}\left(\boldsymbol{J}^{T}\right)=\lambda\left(\begin{array}{cc}
\boldsymbol{R} & 0 \\
0 & \boldsymbol{R}
\end{array}\right)\left(\begin{array}{llll}
0 & 1 & 0 & -1
\end{array}\right)^{T}
$$

allowing one degree of freedom for the design of the effort sharing strategy through the choice of the scalar parameter $\lambda$. Hence, different choices of $\lambda$ will result in different effort sharing policies for forces in the object's $y^{C}$-direction. Each policy will lead to a specific role of the robotic partner by applying a constant share of the desired force input in $y^{C}$ direction. Three policies of a particular physical meaning are discussed below:

\section{- Balanced-effort behavior}

Setting $\lambda=0$ results in the min-norm solution for $\tilde{\boldsymbol{u}}$, hence in a "equitable" sharing policy, which means that the effort is equally shared among human and robot.

\section{- Maximum-robot-effort behavior}

If $\lambda$ is chosen such that the human does not contribute to the object's motion in $y^{C}$-direction, i.e.

$$
\tilde{\boldsymbol{u}}_{1}^{y}=0 \text {, }
$$

which holds true for

$$
\lambda_{1}^{\star}=-\left(\begin{array}{cccc}
0 & 1 & 0 & 0
\end{array}\right)\left(\boldsymbol{J}^{T}\right)^{+} \hat{\boldsymbol{u}},
$$

then the required human force input $\left\|\boldsymbol{u}_{1}\right\|$ is minimized since it is reduced to the translational motion in $x^{C}$ direction and the rotation of the object which simply cannot be accomplished by the robot alone. The robot applies most of the task-relevant forces.

\section{- Minimum-robot-effort behavior}

The least proactive robot behavior w.r.t. effort sharing results from the dual choice to $\lambda_{1}^{\star}$ which is given by

$$
\lambda_{2}^{\star}=-\lambda_{1}^{\star}
$$

which results in the minimum-effort robot assistance. Using this behavior, the robot will only contribute to motion in $x^{C}$-direction and object rotation. The object motion in $y^{C}$-direction will be left to the human partner.

Remark: All choices of $\lambda$ in the interval $\left[\lambda_{1}^{\star} ; \lambda_{2}^{\star}\right]$ are efficient choices in the sense that no counter-acting internal (squeeze) forces are applied to the object.

Besides the choice for $\lambda$ according to (9) or (10), also in order to satisfy (4) one requires the trajectory of $\hat{\boldsymbol{u}}$. The following section shows, how $\hat{\boldsymbol{u}}$ can be obtained for a given task, using a motion-generation and control strategy.

\section{Two-level Hierarchical Architecture}

As previously shown in section II-B.2, an actively contributing robot partner is required for the generalized joint load transport task under environmental constraints. In order to provide these required capabilities the robot must be able to reason about valid (i.e. feasible) paths to the goal in order to shape its own input wrench $\tilde{\boldsymbol{u}}_{2}$. We propose a twolevel hierarchical architecture for planning and control for human-robot cooperative load transport under constraints: On the higher level, a planning algorithm in conjunction with a straightforward interpolation scheme provides a feasible 
trajectory to the goal. Utilizing the system's inverse dynamics we generate the required external input force trajectory $\hat{\boldsymbol{u}}$ for the object. In this first step, we do not consider the joint planning problem. The robot decides on a desired common motion plan which is provided to the human partner during interaction. On the lower level, $\hat{\boldsymbol{u}}$ is decomposed according to the move/squeeze scheme as presented above, and different sharing policies can be applied. Furthermore, a closed-loop impedance control scheme modifies $\hat{\boldsymbol{u}}$ for tolerance towards unexpected human behavior. The proposed architecture is depicted in Fig. 5.

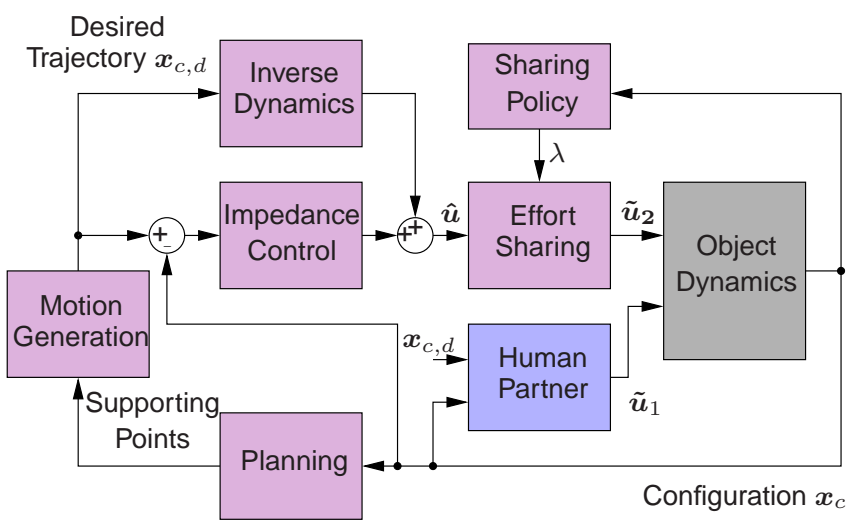

Fig. 5. Two-level hierarchical planning and control architecture colored in magenta. The human partner's interaction behavior is depicted in blue.

\section{A. Planning and Motion Generation}

A classical time-discrete holonomic planning algorithm finds a feasible plan $\pi$ in $\mathcal{C}_{\text {free }}$ to the goal region $\boldsymbol{X}_{G} \in$ $\mathcal{C}_{\text {free }}$, through minimizing the objective function:

$$
L\left(\pi_{K}\right)=\sum_{k=1}^{K} l\left(\boldsymbol{x}_{c, d}^{k}, \hat{\boldsymbol{u}}^{k}\right)+l^{F}\left(\boldsymbol{x}^{F}\right),
$$

where $\mathrm{K}$ denotes the number of stages required to reach the goal and $l\left(\boldsymbol{x}^{k}, \hat{\boldsymbol{u}}^{k}\right)$ is the stage-additive cost of control input $\hat{\boldsymbol{u}}^{k}$ at desired state $\boldsymbol{x}_{c, d}^{k}$. The final term $l^{F}\left(\boldsymbol{x}_{c, d}^{F}\right)$ outside of the sum is defined as $l^{F}\left(\boldsymbol{x}_{c, d}^{F}\right)=0$ if desired final state $\boldsymbol{x}_{c, d}^{F} \in \boldsymbol{X}_{G}$, and $l^{F}\left(\boldsymbol{x}_{c, d}^{F}\right)=\infty$ otherwise.

The path i.e. the plan $\pi$ generated using the above criteria is a series of supporting points. For the joint execution of such plans, a lower level motion generation scheme must be deployed. The straightforward solution is a simple interpolation between the supporting points using a higherorder polynomial interpolation. However, research on haptic cooperation has shown that trajectories of interaction do not necessarily follow the principle of minimum jerk ${ }^{1}$ [18], [19]. As no valid neuro-muscular principle explaining motion generation during interaction could be obtained so far, an interpolation using cubic splines is chosen leading to a smooth, twice continuously differentiable path. Different methods for motion generation are yet to be investigated.

\footnotetext{
${ }^{1}$ jerk denotes the third time derivative of position $\frac{d^{3} \boldsymbol{x}}{d t^{3}}$ which was shown to be an optimization criterion for human free-space motion
}

\section{B. Feedback Interaction Control on Low Level}

The above planning scheme, combined with a refinement method denoted as motion generation produces a feasible desired trajectory for the object configuration $\boldsymbol{x}_{c, d}(t)$. In order to integrate the effort sharing mechanism in an overall planning and control architecture, an object-centered impedancetype control scheme is used to generate the external wrench $\hat{\boldsymbol{u}}$ to be applied on the object. This approach generates a compliant behavior, guaranteeing smooth rising of counteracting wrenches if the human behavior deviates from the expected. The feedback interaction control scheme consists of a feedforward branch, namely the inverse dynamics of the object and a feedback branch closed on the tracking error of the configuration $\boldsymbol{x}_{c}$.

$$
\hat{\boldsymbol{u}}=\boldsymbol{M}_{c} \ddot{\boldsymbol{x}}_{c, d}+\boldsymbol{D}_{c} \dot{\boldsymbol{x}}_{c}+\boldsymbol{K}_{p}\left(\boldsymbol{x}_{c, d}-\boldsymbol{x}_{c}\right)+\boldsymbol{K}_{d}\left(\dot{\boldsymbol{x}}_{c, d}-\dot{\boldsymbol{x}}_{c}\right)
$$

The feedback branch incorporates an impedance control law with $\boldsymbol{K}_{p}$ and $\boldsymbol{K}_{d}$ being the spring and damping gains respectively. To generate the actual control input of the robot $\tilde{\boldsymbol{u}}_{2}$ the solution to the effort sharing problem using (4) is calculated based on $\lambda$. The actual behavior of the effort sharing is defined by the time-invariant policy generating a configuration-dependent $\lambda$, e.g. according to (9) or (10). The effort sharing can be regarded as a selective filter acting on the external wrench $\hat{\boldsymbol{u}}$ dependent on the desired effort sharing behavior.

\section{EXPERIMENTAL EVALUATION}

For experimental evaluation of our approach, we adopt the planar scenario from section III. This experiment aims to show an interdependency between different effort sharing policies on the robot side and the joint tracking performance.

\section{A. Experimental Setup}

In this preliminary study we investigate the effects of three different settings for $\lambda$. Therefore, we asked the subjects to move a virtual bulky object jointly with a virtual robot, visually presented on a display (see Fig. 6). As shown in Fig. 7, the predefined human grasp point is highlighted in magenta, the virtual robot's grasp point is displayed in blue color. Ten right-handed male subjects (age 24-33; mean: 27.7; std: 2.6) were told to track a configuration reference trajectory, the generated motion plan shown in red, together with a virtual robot partner as good as possible. We use the term bulky in order to describe the relative dimensions of the object w.r.t. the environment rather than its absolute expansion. The final configuration was displayed as transparent silhouette.

For generation of experimental data, the virtual robot partner was set to use $\lambda_{1}^{\star}, 0$ and $\lambda_{2}^{\star}$ for $\lambda$ in each trial sequence. The sequences were randomized for each participant. Participants were informed about the virtual robot's behavior before each sequence. Each of the five trials per sequence took $13 \mathrm{~s}$ regardless of the final object configuration. A 5second break allowed the participants to settle between each trial. The first trial of each sequence was discarded from further evaluation. 


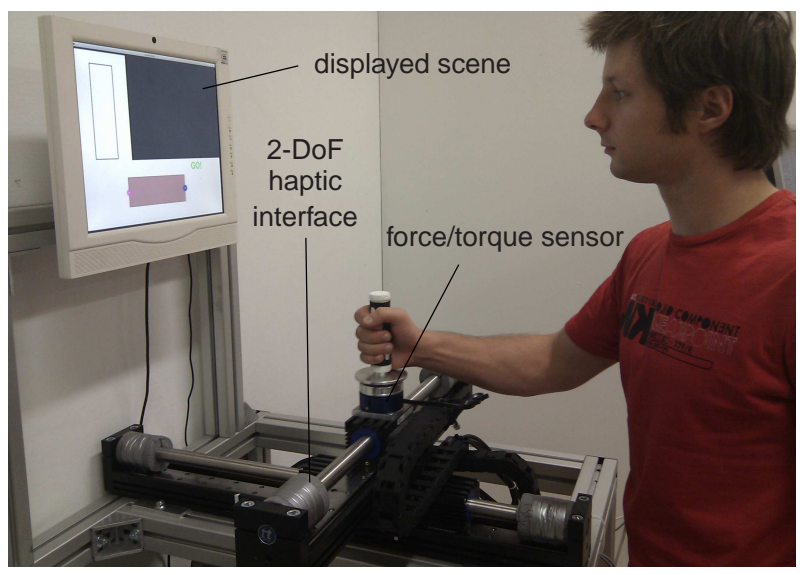

Fig. 6. Experimental Setup.

\section{B. Virtual Robot Partner}

In our implementation we make use of the $P P$ software library [20] which implements a powerful probabilistic roadmap planning algorithm. The generated supporting points are then interpolated using cubic splines, yielding a trajectory $\boldsymbol{x}_{c, d}(\tau), \dot{\boldsymbol{x}}_{c, d}(\tau)$ and $\ddot{\boldsymbol{x}}_{c, d}(\tau)$ where $\tau$ denotes the time variable of the planned trajectory. Implementing a reference adaptive impedance control scheme, we calculate the current value of $\tau$ online by projecting the measured configuration onto the interpolated trajectory, i.e. the current desired input wrench can be calculated at all times. The virtual robot applies the control scheme from Fig. 5 in order to shape its force input to the object.

\section{Hardware Setup}

The user was able to apply forces to the object at the given human grasp point on the opposite side of the virtual robot's grasp point. The force was applied using a haptic interface which returns a position feedback to the human arm. This interface is a two degrees-of-freedom linear-actuated device (ThrustTube) which is attached to a personal computer. A force/torque sensor (JR3) was used to measure the human force input at the free-spinning handle, see Fig. 6 for a picture of the setup.

\section{Software Setup}

The entire control scheme was implemented in MATLAB/Simulink and executed on the Linux Real-Time Application Interface (RTAI) using Matlab's Real-Time Workshop. The control algorithm ran at a frequency of $1 \mathrm{kHz}$. A virtual environment with an object mass of $m=20 \mathrm{~kg}$, rotary inertia of $0.042 \mathrm{kgm}^{2}$ and object dimensions of $0.15 \mathrm{~m}$ by $0.05 \mathrm{~m}$ was physically rendered using Simulink and visualized using Nokia's Qt Class Library [21]. The virtual object's motion was damped by a viscous friction of $120 \mathrm{Ns} / \mathrm{m}$. The visualized scene is presented in Fig. 7. The virtual robot's objectcentered impedance was set to a rotational and translational stiffness of $1.0 \mathrm{Nm} / \mathrm{rad}$ and $100.0 \mathrm{~N} / \mathrm{m}$ and to a rotational and translational damping of $0.6 \mathrm{Nms} / \mathrm{rad}$ and $50.0 \mathrm{Ns} / \mathrm{m}$ respectively.

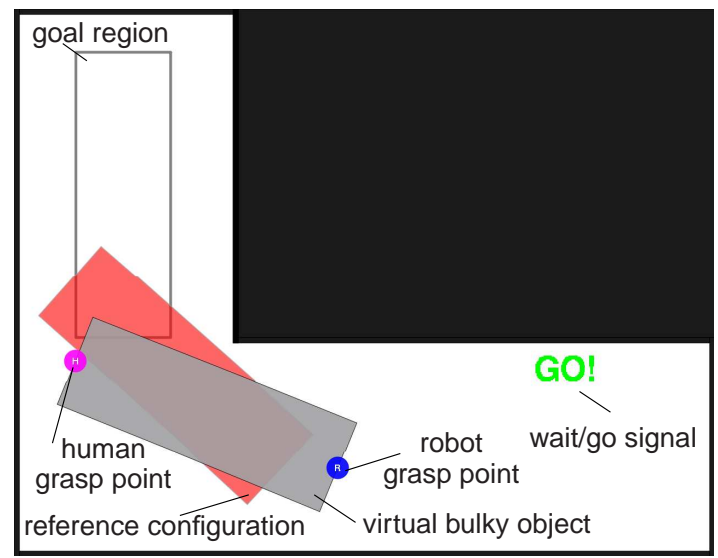

Fig. 7. Annotated screen shot of virtual environment used in the humanrobot experiment.

\section{E. Conditions}

1) Minimum robot effort $\left(\lambda=\lambda_{2}^{\star}\right)$ : In the minimumrobot-effort condition, the robot only contributes to the steering motion but leaves the entire effort in the sharing direction $\left(y^{C}\right)$ to the human.

2) Balanced effort $(\lambda=0)$ : The balanced-effort strategy results in a robot behavior contributing to steering as well as $50 \%$ of the required effort in the sharing direction.

3) Maximum robot effort $\left(\lambda=\lambda_{1}^{\star}\right)$ : In the maximumrobot-effort condition, the robot fulfills the tracking motion in the direction of sharing completely alone. The human force input is only required for steering.

\section{F. Experimental Results}

In Fig. 8 and 9, the root-mean-square force and tracking errors are depicted respectively. Visible trends are discussed in the following.

1) Error Forces: The root-mean-square human force error, calculated by

$$
R M S E_{u_{1}}=\sqrt{\frac{1}{13 s} \int_{0}^{13 s}\left(u_{1, d}(t)-u_{1, m}(t)\right)^{2} d t}
$$

is strongly affected by the robot's effort sharing policy. Erroneous - unnecessary - human force inputs in the direction of effort sharing $\left(y^{C}\right)$ decrease by more than $50 \%$ for the maximum-robot-effort condition in relation to the minimum-robot-effort condition. Forces in perpendicular direction $\left(x^{C}\right)$ seem widely unaffected by the applied sharing policy.

2) Tracking Error: Similarly, the root-mean-square configuration tracking error, calculated by

$$
R M S E_{x_{c}}=\sqrt{\frac{1}{13 s} \int_{0}^{13 s}\left(x_{c, d}(t)-x_{c, m}(t)\right)^{2} d t}
$$

is visibly affected by the robot's effort sharing policy. The tracking performance in the direction of effort sharing $\left(y^{C}\right)$ improves with increasing robot effort. A reciprocal effect can be observed in angular tracking performance, i.e. in $\phi$ direction which deteriorates simultaneously. A potential explanation is the increasing difficulty of mental task modelling 


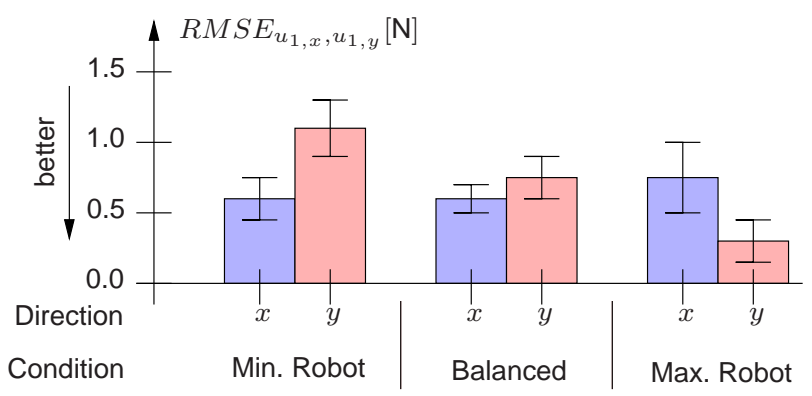

Fig. 8. Mean and standard deviation of the root-mean-square error of the forces applied by the human partner.

as the robot compensates a major share of the object dynamics. No clear conclusion from this experiment can be stated for the $x^{C}$-direction, perpendicular to the direction of effort sharing.

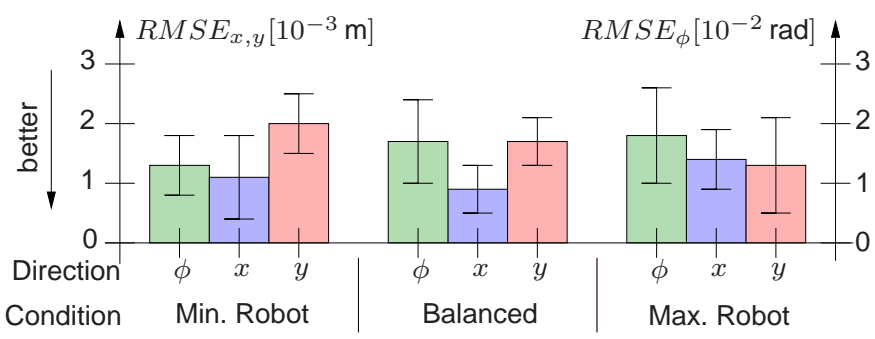

Fig. 9. Mean and standard deviation of the position error.

Based on our experimental investigation we see the following trends:

- In the direction of redundancy-based effort sharing, cooperation quality greatly improves with an increasing degree of robot assistance.

- Tracking performance in the perpendicular i.e. nonshared motion direction remains widely unaffected by the robot's effort sharing strategy.

- The resulting trends of this experiment support a generalization of fundamental findings on human-robot haptic cooperation on point-mass manipulation from the literature [1], [19] to more complex multi-dimensional tasks.

\section{Vi. CONClusion And Future Work}

This paper addresses the problem of effort sharing strategies arising in joint manipulation tasks under environmental constraints. Our approach makes explicit use of the task's geometric, dynamic and environmental properties. The cooperative task is successfully decomposed into a necessary and a voluntary contribution of the cooperating partners respectively. As a result, parameterized effort sharing strategies are derived and discussed. A novel architecture for motion generation and control in joint manipulation embeds these load sharing strategies. A preliminary user study shows that the effort sharing policy has an influence on the cooperative task performance, in particular, that task performance is improved through a higher degree of assistance by the robot, i.e. more proactive behavior.
A detailed evaluation of acceptance by the user as well as an evaluation in higher-dimensional tasks will follow in later studies. The joint planning and plan negotiation problem is subject of our ongoing research.

\section{ACKNOWLEDGEMENTS}

This work is supported in part within the DFG excellence initiative research cluster Cognition for Technical Systems CoTeSys (www. cotesys.org).

\section{REFERENCES}

[1] K. Reed, M. Peshkin, M. Hartmann, J. Patton, P. Vishton, and M. Grabowecky, "Haptic cooperation between people, and between people and machines," in Proc. IEEE/RSJ IROS'06, 2006, pp. 21092114.

[2] K. Reed, M. Peshkin, M. Hartmann, E. Colgate, and J. Patton, "Kinesthetic Interaction," in Proc. IEEE ICORR'05, 2005, pp. 569574.

[3] D. Feth, R. Groten, A. Peer, S. Hirche, and M. Buss, "Performance Related Energy Exchange in Haptic Human-Human Interaction in a Shared Virtual Object Manipulation Task," in Proc. EHS EuroHaptics'09, 2009, pp. 338-343.

[4] P. Evrard and A. Kheddar, "Homotopy Switching Model for Dyad Haptic Interaction in Physical Collaborative Tasks," in Proc. EHS EuroHaptics'09, 2009, pp. 45-50.

[5] O. Oguz, A. Kucukyilmaz, T. Sezgin, and C. Basdogan, "Haptic Negotiation and Role Exchange for Collaboration in Virtual Environments," in Proc. IEEE HAPTICS'10, 2010, pp. 371-378.

[6] G. Montemayor and J. Wen, "Decentralized collaborative load transport by multiple robots," in Proc. IEEE ICRA'05, 2005, pp. 372-377.

[7] T. Wojtara, M. Uchihara, H. Murayama, S. Shimoda, S. Sakai, H. Fujimoto, and H. Kimura, "Human-robot collaboration in precise positioning of a three-dimensional object," Automatica, vol. 45 , no. 2 , pp. $333-342,2009$

[8] T. Takubo, H. Arai, Y. Hayashibara, and K. Tanie, "Human-Robot Cooperative Manipulation Using a Virtual Nonholonomic Constraint," Int. J. Robot. Res., vol. 21, pp. 541-553, 2002.

[9] Y. Hirata and K. Kosuge, "Distributed Robot Helpers Handling a Single Object in Cooperation with Human," in Proc. IEEE ICRA'OO, 2000, pp. 458-463.

[10] K. Kosuge and Y. Hirata, "Human-Robot Interaction," in Proc. IEEE ROBIO'04, 2004, pp. 8-11.

[11] L. Parker, "Distributed Intelligence: Overview of the Field and its Application in Multi-Robot Systems," Jo. Ph. A., vol. 2, pp. 5-14, 2008.

[12] R. Olfati-Saber, A. Fax, and R. Murray, "Consensus and Cooperation in Networked Multi-Agent Systems," Proc. IEEE, vol. 95, pp. 215233, 2007.

[13] A. Lewis and R. Murray, "Configuration Controllability of Simple Mechanical Control Systems," SIAM Review, vol. 41, pp. 555-573, 1999.

[14] W. Heemels and M. Camlibel, "Controllability of Linear Systems with Input and State Constraints," in Proc. IEEE CDC'07, 2007.

[15] E. S. Conkur and R. Buckingham, "Clarifying the definition of redundancy as used in robotics," Robotica, vol. 15, no. 5, pp. 583-586, 1997.

[16] K. Doty, C. Melchiorri, and C. Bonivento, "A Theory of Generalized Inverses Applied to Robotics," Int. J. Robot. Res., vol. 12, pp. 1-19, 1993.

[17] F. Bullo and A. Lewis, Geometric Control of Mechanical Systems, J. Marsden, L. Sirovich, and M. Golubitsky, Eds. Springer, 2004.

[18] T. Flash and N. Hogan, "The coordination of arm movements: An experimentally confirmed mathematical model," J. Neurosci., vol. 5, pp. $1688-1703,1985$.

[19] S. Miossec and A. Kheddar, "Human motion in cooperative tasks: Moving object case study," in Proc. IEEE ROBIO'08, 2008, pp. 15091514.

[20] K. Klasing, "Parallelized sampling-based path planning for treestructured rigid robots," Technische Universität München, Tech. Rep., 2009.

[21] Nokia Corporation. (2010) Qt - A Cross-Platform Application and UI Framework. [Online]. Available: http://qt.nokia.com 